SLAC-TN-05-050

June 2005

\title{
Vertical Arc for ILC Low Emittance Transport
}

\author{
P. Tenenbaum and M. Woodley
}




\section{Vertical Arc for ILC Low Emittance Transport \\ P. Tenenbaum, M. Woodley \\ Stanford Linear Accelerator Center, \\ Stanford, CA 94309, USA}

Abstract

The design and parameters of a vertical arc for the ILC LET are reviewed. 


\title{
Vertical Arc for ILC Low Emittance Transport
}

\author{
P. Tenenbaum, M. Woodley \\ SLAC-ILC-NoTE-0002 \\ June 2, 2005
}

\begin{abstract}
The design and parameters of a vertical arc for the ILC LET are reviewed.
\end{abstract}

\section{Introduction}

A $1 \mathrm{TeV}$ CM ILC which relies upon $30 \mathrm{MV} / \mathrm{m}$ accelerating cavities with a packing fraction of $65 \%$ will require almost $48 \mathrm{~km}$ of main linac, which suggests that the total site length including BDS and bunch compressors will be on the order of $53 \mathrm{~km}$. If built in a laser-straight tunnel with the low-energy ends near the surface, and assuming a perfectly spherical "cue ball" planetary surface with radius $6370 \mathrm{~km}$, the collider halls will necessarily be 55 meters below grade, as shown in the top plot of Figure 1. Such depths would demand extensive use of deep tunneling, which would potentially drive up the cost and difficulty of ILC construction.

An alternate solution is to use discrete vertical arcs at a few locations to allow a "piecewise straight" construction in which the depth of the tunnel below grade does not vary by more than a few meters. This approach is shown schematically in the bottom plot of Figure 1. In this Note we consider the issues for a design with one such vertical arc at the $250 \mathrm{GeV} / \mathrm{c}$ point (ie, midway down the linac for $1 \mathrm{TeV} \mathrm{CM}$ ), and a second arc at the entrance to the BDS (ie, the entire BDS lies in one plane, with vertical arcs at each end).

\section{Saggita of the Proposed Solution}

Figure 2 shows the depth below grade for a $53 \mathrm{~km}$ long ILC in which the accelerator has vertical bends at the approximate center of each linac and outboard of the BDS on each side. In this configuration, the maximum depth variation of any segment is less than $3.5 \mathrm{~m}$, and the depth variation of the BDS is approximately $0.18 \mathrm{~m}$. This is a far more sensible solution than the straight tunnel solution, in which the maximum variation is over 55 meters.

\section{Optics of the Vertical Arc}

Figure 3 shows the optical functions of a vertical arc consisting of five (5) 90-degree FODO cells interleaved with bend magnets. The optics achieves a zero dispersion at entrance and exit by using the dispersion matching scheme of Keil [1]. The system as shown is designed to achieve a 1.309 mrad total bend angle, which corresponds to the required bend between the second half of the linac and the BDS, assuming system lengths of $11.9 \mathrm{~km}$ for the former and $2.37 \mathrm{~km}$ for the latter. System total length is 160 meters. Table 1 shows the key parameters for the magnets in the system.

As shown in Figure 3, the vertical arc does not incorporate any dispersion correction quadrupoles. The vertical dispersion can be corrected by adjusting the strengths of some of the main FODO quads in $-I$ pairs (ie, change a quad at a given point and perform an equal-and-opposite change of another quad which is exactly 2 cells away). Because the optics contains 5 cells, it is possible to correct 
dispersion in both betatron phases without interleaving corrections. Correction of horizontal dispersion would require insertion of skew quadrupoles, which can easily be accomplished at the cost of a slight increase in system length. Given that the horizontal dispersion must be coupled out of the vertical plane by bend or quad rotation errors, that the horizontal emittance is much larger than the vertical, and that the energy spread will be relatively small, it may be practical to simply forego horizontal dispersion correction entirely.

Table 1: Parameters of magnets in the $1.3 \mathrm{mrad}$ vertical arc between the end of the linac and the BDS.

\begin{tabular}{|c|c||c|c|c|c|}
\hline Name & Type & Length $[\mathrm{m}]$ & Pole Field [T] & Aperture Radius [mm] & Count \\
\hline \hline VQF & Quad & 1.0 & 0.85 & 20 & 20 \\
\hline VQD & Quad & 1.0 & 0.85 & 20 & 20 \\
\hline VB1 & Bend & 2.0 & 0.023 & 20 & 16 \\
\hline VB2 & Bend & 2.0 & 0.023 & 20 & 16 \\
\hline VB & Bend & 2.0 & 0.045 & 20 & 8 \\
\hline
\end{tabular}

\section{Synchrotron Radiation Issues}

The energy loss per particle from synchrotron radiation is given by [2]:

$$
\Delta E=\frac{2}{3} \frac{r_{e} E^{4}}{\left(m_{e} c^{2}\right)^{3}} I_{2}
$$

where $r_{e}$ is the classical electron radius of $2.8 \times 10^{-15}$ meters and $I_{2}$ is the second synchrotron radiation integral, $I_{2} \equiv \int d s / \rho^{2}$. For the system shown in Figure $3, I_{2}=2.38 \times 10^{-7} \mathrm{~m}^{-1}$, yielding an energy loss per electron of $208 \mathrm{MeV}$ for $500 \mathrm{GeV} / \mathrm{c}$ beams. For $500 \mathrm{GeV} / \mathrm{c}$ beams with 22.6 MW beam power, the power dissipated in the vertical arc is $9.4 \mathrm{~kW}$.

The growth in normalized emittance from synchrotron radiation is given by:

$$
\Delta \gamma \epsilon=40.43 \mathrm{~nm} E^{6}\left[\mathrm{GeV}^{6}\right] I_{5}\left[\mathrm{~m}^{-1}\right],
$$

where $I_{5}$ is the fifth synchrotron radiation integral, $I_{5} \equiv \int d s\left(\gamma \eta^{2}+2 \alpha \eta \eta^{\prime}+\beta \eta^{\prime 2}\right) /|\rho|^{3}$. For the system shown in Figure 3, $I_{5}=7.85 \times 10^{-19} \mathrm{~m}^{-1}$, yielding a normalized vertical emittance growth at $500 \mathrm{GeV} / \mathrm{c}$ beam energy of $0.50 \mathrm{~nm}$, which is $2.5 \%$ of the damping ring extracted emittance specification of $20 \mathrm{~nm}$.

\section{$5 \quad$ Energy Scaling}

In the previous Section we considered the energy loss and emittance growth for $500 \mathrm{GeV} / \mathrm{c}$ beams passing through the $1.3 \mathrm{mrad}$ vertical arc from the main linac into the BDS. The vertical arc required at the $250 \mathrm{GeV} / \mathrm{c}$ point in the linac has a larger total bend angle of approximately 2.1 mrad. This is compensated by the much lower beam energy. Since mean energy loss scales as $E^{4} \theta^{2}$, and normalized emittance growth scales as $E^{6} \theta^{5}$, we conclude that we can use the same optics with scaled-up bend strengths at the $250 \mathrm{GeV} / \mathrm{c}$ point and expect negligible energy loss and emittance growth. 
More problematic is the case of energy upgrade, for example to $3 \mathrm{TeV} \mathrm{CM}$ (or equivalently $1.5 \mathrm{TeV} / \mathrm{c}$ final beam energy). The arc at the end of the linac would generate $16 \mathrm{GeV}$ energy loss and normalized emittance growth of $364 \mathrm{~nm}$. The emittance growth is certainly unacceptable, especially if the normalized emittance is to be reduced to $5 \mathrm{~nm}$ as part of the energy upgrade, as some parameter sets propose; the energy loss of $1 \%$ is likely also unacceptable, as it would certainly entail dumping on the order of $100 \mathrm{~kW}$ in the arcs.

In the case of upgrades to such high energies, we note that the current packing fraction of bend magnets in the arc is only about $50 \%$. One possible scenario for upgrade is to replace the arc design presented here with an arc which uses a dense array of combined function bend magnets (essentially quadrupole magnets with mm-scale intentional offsets with respect to the survey line), and possibly to slightly extend the length of the arc into the linac and BDS. Given the rapid scaling of synchrotron radiation effects with bend radius and focusing, an acceptable solution for $3 \mathrm{TeV}$ CM should be achievable.

\section{References}

[1] E. Keil, CERN 77-13 (1977).

[2] R. H. Helm, M. J. Lee, P.L. Morton, "Evaluation of Synchrotron Radiation Integrals" (1973). 


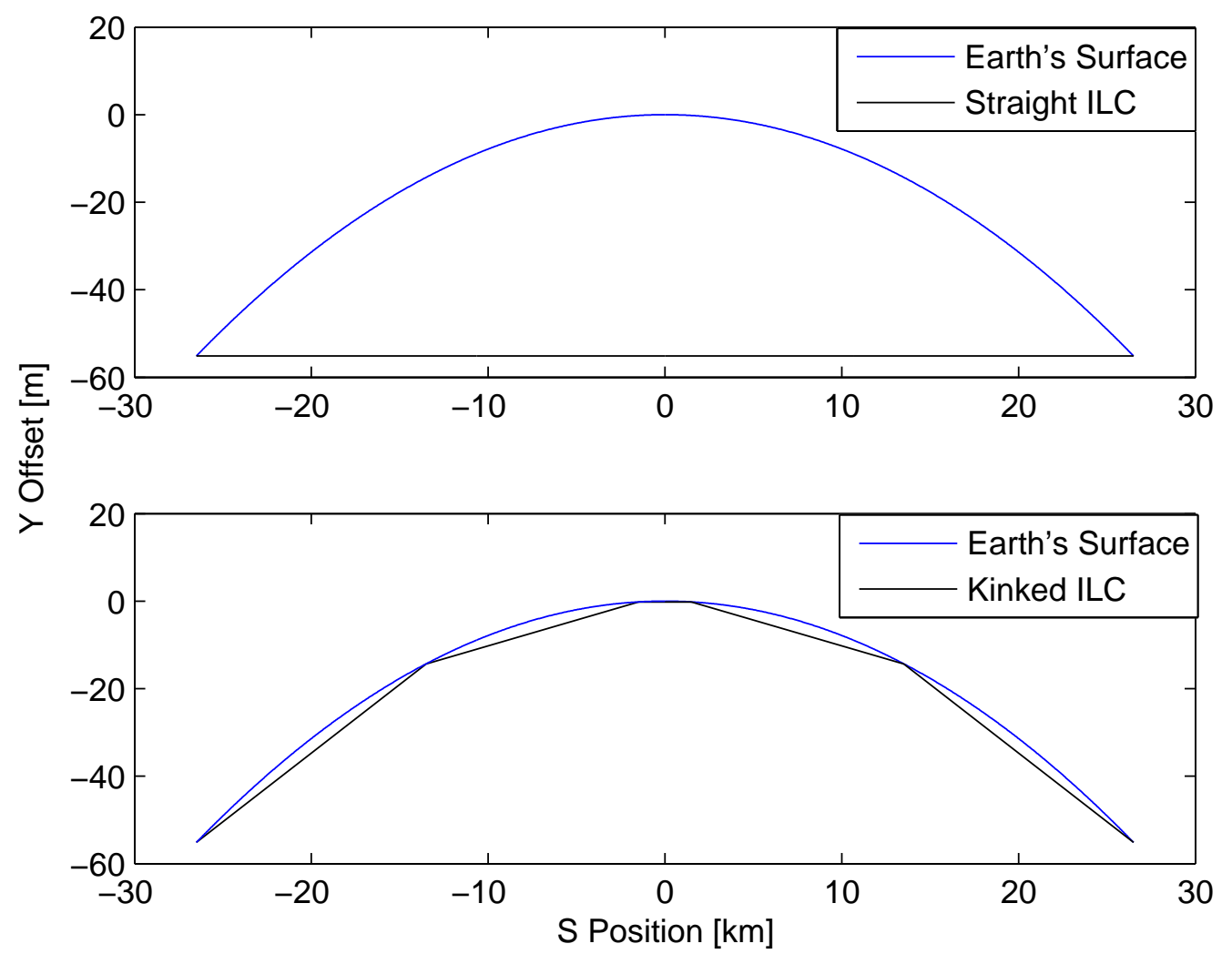

Figure 1: Two possible configurations for a $53 \mathrm{~km}$ ILC site. Top: a perfectly straight tunnel running under the curved surface of the Earth. Bottom: A piecewise straight configuration as described in the text. 


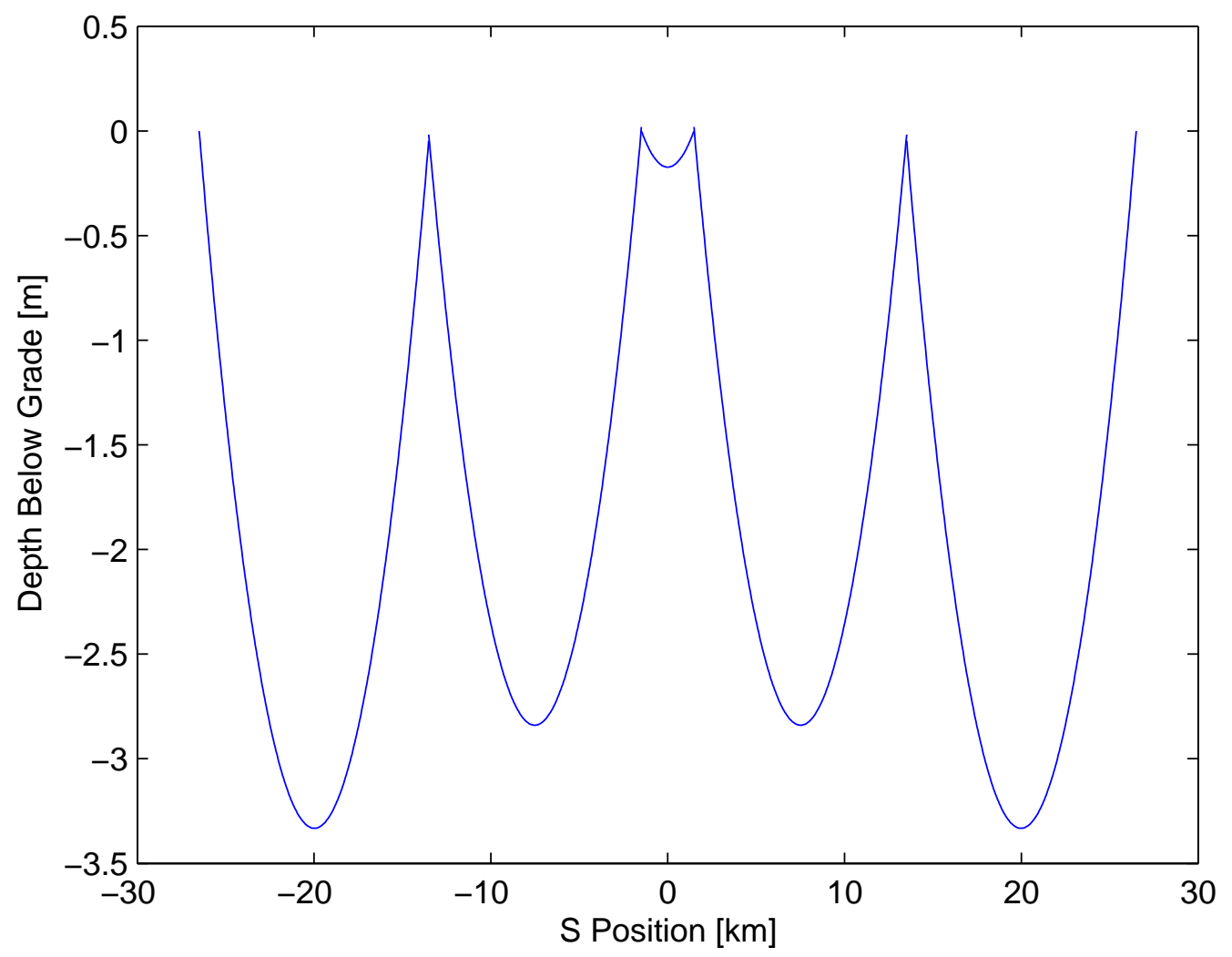

Figure 2: Depth variation for the piecewise-straight ILC as described in the text. 


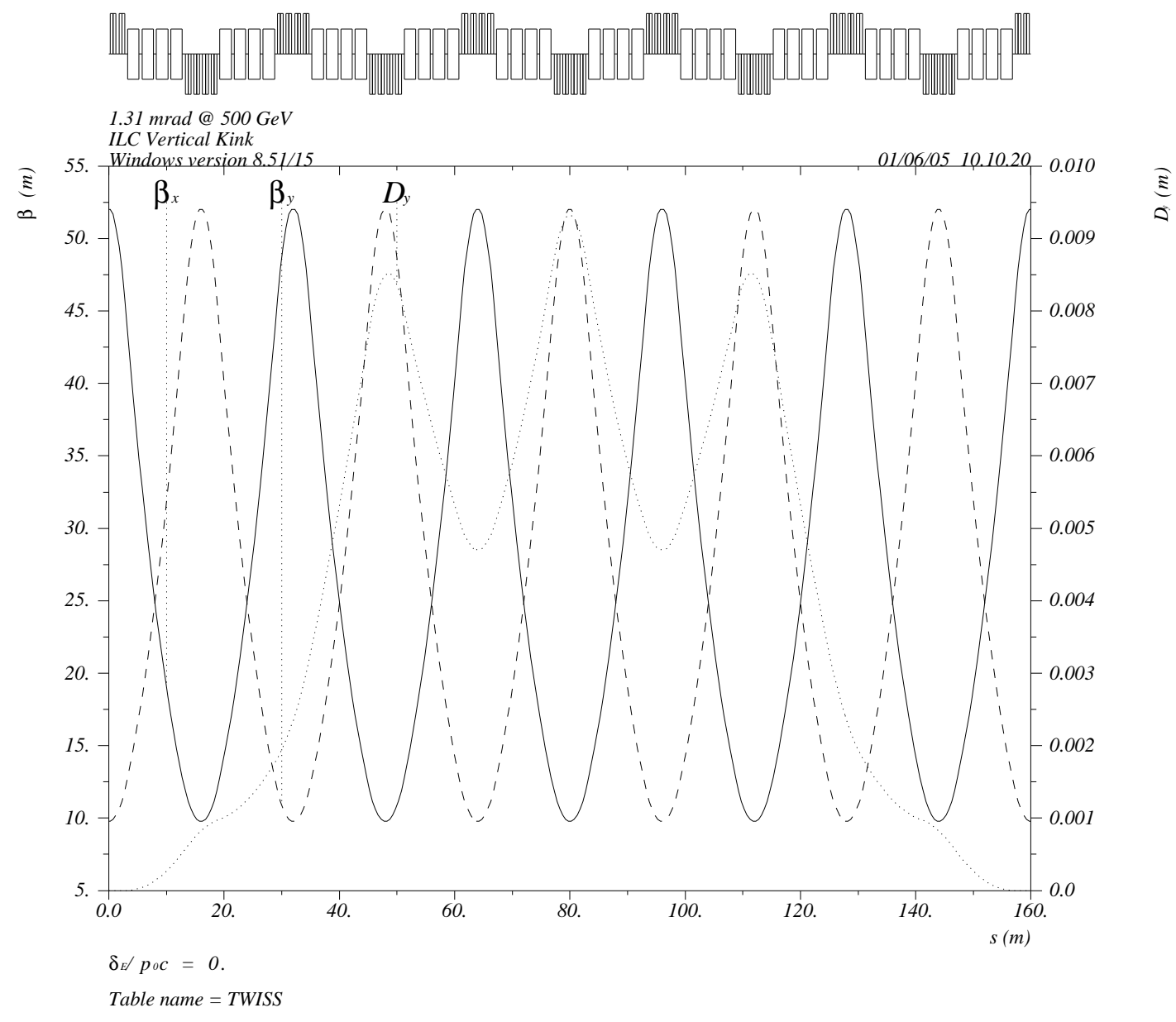

Figure 3: Optics function of the $1.3 \mathrm{mrad}$ vertical arc required between the end of the linac and the entry to the BDS. 